\title{
Investigating whether adverse prenatal and perinatal events are associated with non-clinical psychotic symptoms at age 12 years in the ALSPAC birth cohort
}

\author{
S. Zammit*, D. Odd, J. Horwood, A. Thompson, K. Thomas, P. Menezes, D. Gunnell, \\ C. Hollis, D. Wolke, G. Lewis and G. Harrison
}

Academic Unit of Psychiatry, University of Bristol, UK

Background. Non-clinical psychosis-like symptoms (PLIKS) occur in about $15 \%$ of the population. It is not clear whether adverse events during early development alter the risk of developing PLIKS. We aimed to examine whether maternal infection, diabetes or pre-eclampsia during pregnancy, gestational age, perinatal cardiopulmonary resuscitation or 5-min Apgar score were associated with development of psychotic symptoms during early adolescence.

Method. A longitudinal study of 6356 12-year-old adolescents who completed a semi-structured interview for psychotic symptoms in the Avon Longitudinal Study of Parents and Children (ALSPAC) birth cohort. Prenatal and perinatal data were obtained from obstetric records and maternal questionnaires completed during pregnancy.

Results. The presence of definite psychotic symptoms was associated with maternal infection during pregnancy [adjusted odds ratio (OR) 1.44, 95\% confidence interval (CI) 1.11-1.86, $p=0.006$ ], maternal diabetes (adjusted OR 3.43, 95\% CI 1.14-10.36, $p=0.029$ ), need for resuscitation (adjusted OR 1.50, 95\% CI 0.97-2.31, $p=0.065$ ) and 5-min Apgar score (adjusted OR per unit decrease 1.30, 95\% CI 1.12-1.50, $p<0.001$ ). None of these associations were mediated by childhood IQ score. Most associations persisted, but were less strong, when including suspected symptoms as part of the outcome. There was no association between PLIKS and gestational age or pre-eclampsia.

Conclusions. Adverse events during early development may lead to an increased risk of developing PLIKS. Although the status of PLIKS in relation to clinical disorders such as schizophrenia is not clear, the similarity between these results and findings reported for schizophrenia indicates that future studies of PLIKS may help us to understand how psychotic experiences and clinical disorders develop throughout the life-course.

Received 19 June 2008; Revised 14 November 2008; Accepted 11 December 2008; First published online 12 February 2009

Key words: Cohort study, obstetric complications, pregnancy, psychotic symptoms.

\section{Introduction}

About $15 \%$ of the population report experiencing delusions or hallucinations (Eaton et al. 1991; Poulton et al. 2000; van Os et al. 2001; Johns et al. 2004; Wiles et al. 2006), although the prevalence of clinical psychotic disorders is much lower (Kendler et al. 1996; Perala et al. 2007). It is not clear whether these relatively common psychotic experiences represent an early expression of neurodevelopmental pathological processes that lead to schizophrenia, or whether they simply reflect common variation in the way individuals cognitively appraise, and describe, their

* Address for correspondence: Dr S. Zammit, Department of Psychological Medicine, School of Medicine, Cardiff University, Heath Park, Cardiff CF14 4XN, Wales, UK.

(Email: zammits@Cardiff.ac.uk) surrounding environment, with little or no implications for health.

Although the body of evidence is not strong, results from the Dunedin study (Poulton et al. 2000) and the Netherlands Mental Health Survey and Incidence Study (NEMESIS; Hanssen et al. 2005) cohorts suggest that people experiencing such symptoms may be at increased risk of developing clinically important psychotic disorders later in life. Studying psychosis-like symptoms (PLIKS) may increase our understanding of the development of psychotic experiences, and potentially help to elucidate aetiological mechanisms underlying schizophrenia.

The neurodevelopmental model of schizophrenia postulates that neural insults from embryonic development through childhood and adolescence all play a causal role in the onset of this disorder. For example, maternal exposure to famine (Susser et al. 1996; 
St Clair et al. 2005) or influenza (Brown et al. 2004; Byrne et al. 2007) and also other prenatal and perinatal complications (see review by Cannon et al. 2002) have been associated with increased risk of schizophrenia in the offspring. A cross-sectional study of adolescents reported no association between psychotic symptoms and composite measures of pregnancy and birth complications, as recalled by the mothers (Spauwen et al. 2004). However, there have been no longitudinal studies to date that we are aware of that have examined whether specific, adverse prenatal or perinatal events exposures are associated with the development of non-clinical psychotic symptoms.

\section{Method}

Sample

This study examined data from 6356 children from the Avon Longitudinal Study of Parents and Children (ALSPAC) cohort who participated in the PLIKS semistructured interview (PLIKSi) (Horwood et al. 2008) at age 12 years (data restricted to one child per nuclear family). The initial ALSPAC cohort (www.alspac.bris. ac.uk) consisted of 14062 children born to residents of the former Avon Health Authority area who had an expected date of delivery between 1 April 1991 and 31 December 1992. The cohort was set up to examine genetic and environmental determinants of health and development (Golding et al. 2001). The parents have completed regular postal questionnaires about all aspects of their child's health and development since birth. The children have attended annual assessment clinics since age 7 years. Because of attrition and wave non-response, sample sizes in the analyses differ according to exposures and datasets examined (see Results and tables).

\section{Measures}

\section{Outcomes}

The PLIKSi covers past 6-month occurrence of hallucinations (visual and auditory); delusions (delusions of being spied on, persecution, thoughts being read, reference, control, grandiose ability and other unspecified delusions); and experiences of thought interference (thought broadcasting, insertion and withdrawal). For these 12 core items, seven screening (stem) questions were derived from the Diagnostic Interview Schedule for Children version IV (DISCIV; Shaffer et al. 2000) and five questions from the Schedules for Clinical Assessment in Neuropsychiatry (SCAN) version 2.0 (WHO, 1994) modified slightly after piloting [further details available at Academic Unit of Psychiatry, University of Bristol (http:// www.bris.ac.uk/psychiatry/research/psychotic.html)]. Coding of all items followed the glossary definitions and rating rules for SCAN, and clinical crossquestioning and probing by psychologists trained in using the PLIKSi was used to establish the presence or absence of symptoms. Interviewers rated symptoms as either not present, suspected or definitely present. Unclear responses after probing were always 'rated down', and symptoms were only rated as definite when a credible example was provided. We included symptoms in our analyses only if they were not attributable to effects of sleep, fever or substance use, consistent with the approach of classification systems for diagnosis of functional psychotic disorders. The average $\kappa$ value for inter-rater reliability was 0.72 .

We examined two primary PLIKS outcomes: $(a)$ the presence of any suspected or definite symptoms, and (b) a narrower outcome of definite symptoms only. As secondary analyses, we also examined associations with more frequently occurring symptoms (definite symptoms occurring $\geqslant$ monthly), and with symptoms that may be more characteristic of schizophrenia (any suspected or definite 'bizarre' PLIKS). These symptoms, accorded greater weighting in both DSM-IV and ICD-10 criteria for schizophrenia, included either third-person auditory hallucinations, delusions of control, or delusions of thought broadcast, insertion or withdrawal.

\section{Exposures (a)}

In the main dataset we examined the following pregnancy-related exposures: (i) maternal influenza or any other infections, (ii) need for resuscitation, (iii) 5-min Apgar score, and (iv) gestational age at birth.

Data on prenatal exposure to influenza or other infections were obtained from self-report postal questionnaires completed by the mother at 18 and 32 weeks of pregnancy, and 2 months postnatally. We examined associations with these exposures at any time during pregnancy, and also examined whether effects were different according to trimester of exposure.

Information on admission, resuscitation and perinatal well-being was retrieved from computerized records of all infants born in the two main maternity hospitals in the region ( $92 \%$ of the cohort). Our primary measure of hypoxia was resuscitation, defined as either positive pressure respiratory support (using a face mask or endotracheal tube) or cardiac compressions. Receipt of ambient oxygen alone was not considered to be a marker of clinical hypoxia, and these infants were included in the non-resuscitation group. In addition to comparing infants who were or were not resuscitated, we examined whether associations were stronger for children who received 
resuscitation and (a) were admitted to a neonatal unit, and $(b)$ also developed signs of encephalopathy (defined as the presence of seizures, jitteriness, a highpitched cry, hypo- or hypertonia, or hyper-reflexia during admission). Data on 5-min Apgar score were examined as a marker of perinatal well-being (scores ranging from 0 to 10 , with 10 being the best outcome). Gestational age was analysed both as continuous (weeks) and categorical [pre-term ( $\leqslant 36$ weeks), normal term (37-42 weeks), post-term ( $>42$ weeks)] data.

\section{Exposures (b)}

We also conducted a nested case-control study to examine whether maternal diabetes or pre-eclampsia was associated with PLIKS. Information on these two exposures was available only after manual retrieval and examination of obstetric records. As resources were limited, this was done for all adolescents who reported PLIKS and a random $20 \%$ of those without PLIKS on interview. Data were extracted blind to PLIKS status. We examined PLIKS associations with (a) either a clinician diagnosis of diabetes in the obstetric records or self-reported diabetes from a questionnaire at 12 weeks gestation, and $(b)$ poorly controlled diabetes, defined as above but with the additional presence of either birthweight $>90$ th percentile or maternal glycosuria recorded on $\geqslant 3$ antenatal visits. For pre-eclampsia, we examined associations with (a) maternal pre-eclampsia (defined as systolic blood pressure $\geqslant 140 \mathrm{mmHg}$ or diastolic $\geqslant 90 \mathrm{mmHg}$, with proteinuria ( $>$ trace), on $\geqslant 2$ antenatal visits), and (b) pre-eclampsia with intra-uterine growth restriction (IUGR) (as above but with the additional presence of birthweight $<10$ th percentile).

\section{Confounders}

Potential confounders were selected a priori on the basis of previous reports in the literature of their association with pregnancy or birth complications and with psychosis. To examine the potential confounding impact of multiple family risk factors, a Family Adversity Index (FAI) was used (Bowen et al. 2005). The FAI consists of 18 items taken from questionnaires that were administered during pregnancy. The index was based on a series of measures describing various aspects of family functioning covering early parenthood (maternal age $<20$ years at first childbirth), housing adequacy, financial difficulties, parent educational qualifications, family size, social support, maternal relationship with partner, maternal affective disorder, parental substance abuse, and involvement with crime. If adversity was present this was rated as 1 and then totalled across the 18 items.
Other confounders adjusted for include urban/rural index at birth (urban/town, village/hamlet), maternal age, maternal use of prescribed medication (analgesics or hypnotics), maternal smoking during pregnancy, and maternal depression during pregnancy [Edinburgh Postnatal Depression Scale (EPDS); Cox et al. 1987]. For maternal diabetes we also adjusted for maternal body mass index (BMI).

We considered child total IQ score at age 8, from the Wechsler Intelligence Scale for Children-Third Edition (WISC-III; Wechsler, 1991), as a potential mediator of any relationship between prenatal or perinatal exposures and development of PLIKS (i.e. lying on the causal pathway). We also considered birthweight (as a marker of chronic in-utero adversity) as a potential mediator for prenatal exposures, as lower birthweight was found to be associated with PLIKS at age 12 in this cohort (Thomas et al. in press).

\section{Ethical approval}

Ethical approval for the study was obtained from the ALSPAC Law and Ethics Committee and the Local Research Ethics Committees.

\section{Statistical analysis}

Logistic regression was used to calculate odds ratios (ORs) and 95\% confidence intervals (CIs) for PLIKS given the prenatal and perinatal exposures. Examination of whether a non-linear relationship (within the logistic model) between weeks of gestation and PLIKS provided a better fit for the data was made by inclusion of quadratic terms and use of likelihood ratio tests (LRTs) to compare different models. All analyses, apart from those examining gestational age, were restricted to term births ( $>36$ and $<43$ weeks' gestation).

\section{Missing data}

Attrition is a problem common to all large-scale longitudinal studies (Plewis et al. 2004; Callaway et al. 2007). To examine whether missing data may have biased our results, we conducted sensitivity analyses using multiple imputations by chained equations (Raghunathan et al. 2001; Royston, 2004). We used the ice command in Stata version 9 (Stata Corporation, College Station, TX, USA) to impute confounder and outcome missing data. Approximately 50 variables relating to parental sociodemographic factors and child emotional, social and behavioural characteristics were used to impute the missing data. Ten cycles of regression were carried out and 25 datasets imputed. 
Table 1. Number (\%) of children within exposure category with confounder present ${ }^{\text {a }}$

\begin{tabular}{|c|c|c|c|c|c|c|c|}
\hline & Male & $\begin{array}{l}\text { FAI }>90 \text { th } \\
\text { percentile }\end{array}$ & $\begin{array}{l}\text { Mother's age } \\
>30 \text { years }\end{array}$ & $\begin{array}{l}\text { Maternal } \\
\text { depression } \\
(\text { EPDS } \geqslant 15)\end{array}$ & $\begin{array}{l}\text { Medication } \\
\text { during } \\
\text { pregnancy }\end{array}$ & $\begin{array}{l}\text { Maternal } \\
\text { smoking in } \\
\text { pregnancy }\end{array}$ & Rural birth \\
\hline \multicolumn{8}{|c|}{ Infection in pregnancy } \\
\hline No & $2762(51.2)$ & $413(7.7)$ & $1702(31.6)$ & $202(3.8)$ & $3102(57.6)$ & $1147(21.3)$ & $310(5.8)$ \\
\hline Yes & $3061(51.8)$ & $731(12.6)$ & $1862(31.5)$ & $467(8.1)$ & $4021(70.2)$ & $1774(30.0)$ & $326(5.6)$ \\
\hline \multicolumn{8}{|c|}{ Diabetes in pregnancy } \\
\hline No & $1705(49.9)$ & $344(10.6)$ & $1010(29.6)$ & $237(7.3)$ & $2094(65.2)$ & $934(27.7)$ & $164(4.9)$ \\
\hline Yes & $23(53.5)$ & 5 (11.6) & $15(34.9)$ & $4(9.3)$ & 33 (78.6) & $6(14.0)$ & $0(0)$ \\
\hline \multicolumn{8}{|c|}{ Pre-eclampsia in pregnancy } \\
\hline No & $1493(49.8)$ & $298(10.4)$ & 891 (29.7) & $207(7.3)$ & $1860(65.8)$ & $812(27.4)$ & $144(4.9)$ \\
\hline Yes & $51(60.7)$ & $8(10.5)$ & $32(38.1)$ & $13(17.6)$ & $45(63.4)$ & $19(23.2)$ & $2(2.4)$ \\
\hline \multicolumn{8}{|c|}{ Preterm birth } \\
\hline No & $6698(51.3)$ & $1265(10.3)$ & $3924(30.1)$ & $777(6.4)$ & $7860(65.1)$ & 3555 (27.6) & $709(5.5)$ \\
\hline Yes & $465(58.1)$ & $81(11.0)$ & $214(26.8)$ & $72(9.8)$ & $465(66.4)$ & $242(30.7)$ & $52(6.5)$ \\
\hline \multicolumn{8}{|c|}{ Resuscitated } \\
\hline No & 5853 (51.2) & $1074(10.0)$ & 3493 (30.6) & $683(6.5)$ & 6865 (65.1) & 3055 (27.1) & $611(5.4)$ \\
\hline Yes & $625(55.4)$ & 119 (11.3) & $317(28.2)$ & 67 (6.5) & $680(66.2)$ & 318 (28.6) & 48 (4.3) \\
\hline \multicolumn{8}{|c|}{ Apgar score $<6$} \\
\hline No & $6464(51.5)$ & 1189 (10.1) & $3822(30.5)$ & 755 (6.5) & 7541 (65.1) & 3374 (27.3) & $672(5.4)$ \\
\hline Yes & 94 (59.9) & $22(15.3)$ & $32(20.5)$ & 14 (9.6) & $98(69.5)$ & 57 (36.8) & $6(3.9)$ \\
\hline
\end{tabular}

FAI, Family Adversity Index; EPDS, Edinburgh Postnatal Depression Scale.

${ }^{a}$ Confounding variables were dichotomized for the purpose of this table only and not for analyses.

\section{Results}

There were 734 children $(11.6 \%$ of those interviewed; $95 \%$ CI 10.8-12.4) rated as having suspected or definite PILKS not attributable to fever or sleep. Of these, $300(4.7 \%$ of those interviewed) had definite symptoms. A summary of potential confounders in relation to the exposures examined is presented in Table 1.

\section{Infection during pregnancy}

There were 5379 women with data available for infection during pregnancy, confounders, and PLIKS data in their offspring. Of these, $2582(48.0 \%)$ reported having had any infection, and $863(16.0 \%)$ specifically reported having influenza. There was no evidence that having influenza at any time during pregnancy was associated more, or less, strongly than having other, non-influenza, infections (Table 2). We therefore present results here for any infection (influenza and noninfluenza infections combined together).

Having any infection at any time during pregnancy was associated with any suspected or definite PLIKS in the offspring (adjusted OR 1.31, 95\% CI 1.10-1.56, $p=0.002$ ). This estimate was not substantially different when we examined definite PLIKS as the outcome (adjusted OR 1.44, 95\% CI 1.11-1.86, $p=0.006$ ). Further adjusting for birthweight or childhood IQ as possible mediators for this association had minimal effect on these results.

We also examined the effects of infection during specific trimesters. There were 658 women who had had an infection only during their first trimester, 471 only during their second trimester, and 335 only during their third trimester. Estimates of association with PLIKS were larger for early pregnancy exposure to infection (first trimester only compared to no infection, adjusted OR 1.41, 95\% CI 1.09-1.83; second trimester only, OR $1.36,95 \%$ CI 1.01-1.82; third trimester only, OR $1.16,95 \%$ CI 0.81-1.66). However, the CIs for these estimates overlapped substantially, and there was no statistical evidence of a greater risk of PLIKS with first-trimester compared to third-trimester exposure (adjusted OR 1.26, 95\% CI 0.85-1.87, $p=0.441$ ).

\section{Resuscitation}

There were 5197 children with data available for PLIKS, confounders and resuscitation status. Of these, $390(7.5 \%)$ received positive pressure ventilation or cardiac compressions. Infants who were resuscitated had an increased risk of developing any suspected or definite PLIKS (adjusted OR 1.34, 95\% CI 1.00-1.81, $p=0.053$ ). This estimate was not substantially different 
Table 2. Crude and adjusted odds ratios of PLIKS outcomes for prenatal and perinatal exposures (full sampla ${ }^{\mathrm{a}}$ )

\begin{tabular}{|c|c|c|c|c|c|c|c|c|c|c|}
\hline & \multirow[b]{2}{*}{$\begin{array}{l}\text { No. in } \\
\text { sample }\end{array}$} & \multirow[b]{2}{*}{$\begin{array}{l}\text { Exposure, } \\
\text { no PLIKS }\end{array}$} & \multicolumn{4}{|c|}{ Suspected or definite PLIKS } & \multicolumn{4}{|c|}{ Definite PLIKS } \\
\hline & & & $\begin{array}{l}\text { Exposure, } \\
\text { with PLIKS }\end{array}$ & $\begin{array}{l}\text { Crude OR } \\
(95 \% \mathrm{CI})\end{array}$ & $\begin{array}{l}\text { Adjusted OR }{ }^{b} \\
(95 \% \text { CI })\end{array}$ & $p$ value & $\begin{array}{l}\text { Exposure, } \\
\text { with PLIKS }\end{array}$ & $\begin{array}{l}\text { Crude OR } \\
(95 \% \mathrm{CI})\end{array}$ & $\begin{array}{l}\text { Adjusted OR } \\
(95 \% \text { CI })\end{array}$ & $p$ value \\
\hline \multicolumn{11}{|l|}{ Infection in pregnancy } \\
\hline Influenza & & 739 & 124 & $1.56(1.24-1.96)$ & $1.39(1.10-1.76)$ & & 47 & $1.45(1.02-2.06)$ & $1.22(0.85-1.76)$ & \\
\hline Non-influenza & & 1500 & 219 & $1.36(1.12-1.64)$ & $1.27(1.05-1.54)$ & & 108 & $1.68(1.28-2.22)$ & $1.55(1.17-2.04)$ & \\
\hline Any infection & 5379 & 2239 & 343 & $1.42(1.20-1.68)$ & $1.31(1.10-1.56)$ & 0.002 & 155 & $1.60(1.25-2.07)$ & $1.44(1.11-1.86)$ & 0.006 \\
\hline Gestation (per week $\uparrow$ ) & 6004 & - & - & $1.01(0.97-1.05)$ & $1.01(0.96-1.05)$ & 0.736 & - & $1.05(0.98-1.13)$ & $1.05(0.98-1.13)$ & 0.176 \\
\hline \multicolumn{11}{|l|}{ Resuscitation status } \\
\hline No resuscitation & & 4273 & 534 & 1.0 & 1.0 & & 213 & 1.0 & 1.0 & \\
\hline Resuscitation, not admitted & & 292 & 46 & $1.26(0.91-1.74)$ & $1.27(0.92-1.76)$ & & 21 & $1.43(0.90-2.27)$ & $1.48(0.93-2.35)$ & \\
\hline $\begin{array}{l}\text { Resuscitation, admitted - no } \\
\text { symptoms }\end{array}$ & & 25 & 6 & $1.92(0.78-4.70)$ & $1.84(0.74-4.54)$ & & 3 & $2.31(0.70-7.66)$ & $2.14(0.63-7.28)$ & \\
\hline $\begin{array}{l}\text { Resuscitation, admitted - and } \\
\text { encephalopathy }\end{array}$ & & 17 & 4 & $1.88(0.63-5.62)$ & $1.82(0.60-5.48)$ & & 1 & $1.08(0.14-8.07)$ & $0.98(0.13-7.35)$ & \\
\hline Any resuscitation versus none & 5197 & 334 & 56 & $1.34(1.00-1.81)$ & $1.34(1.00-1.81)$ & 0.053 & 25 & $1.48(0.96-2.27)$ & $1.50(0.97-2.31)$ & 0.065 \\
\hline Apgar score (per 1 patient $\downarrow$ ) & 5262 & - & - & $1.06(0.96-1.21)$ & $1.06(0.95-1.19)$ & 0.292 & - & $1.31(1.14-1.51)$ & $1.30(1.12-1.50)$ & $<0.001$ \\
\hline
\end{tabular}

PLIKS, Psychosis-like symptoms; OR, odds ratio; CI, confidence interval.

a Analyses restricted to dataset with no missing data for confounding factors.

${ }^{\mathrm{b}}$ Adjusted for Family Adversity Index (FAI), sex, urban/rural birth, maternal age, maternal smoking, maternal depression, and medication use during pregnancy. 
when we examined definite PLIKS as the outcome (adjusted OR 1.50, 95\% CI 0.97-2.31, $p=0.065$ ). Further adjusting for childhood IQ as a possible mediator for this association had minimal effect on these results.

Of the 390 infants resuscitated, 52 were additionally admitted to a neonatal unit and 21 of these developed signs of encephalopathy. The estimates of association with any suspected or definite PLIKS were slightly larger for infants who were resuscitated and required admission to a neonatal unit irrespective of whether they developed encephalopathy (adjusted OR 1.82, $95 \%$ CI $0.60-5.48$ ) or not (adjusted OR 1.84, 95\% CI 0.74-4.54), compared to children who were resuscitated but did not require admission (adjusted OR 1.27, 95\% CI 0.92-1.76). However, these estimates were based on small numbers of events and the CIs overlapped substantially.

\section{5-min Apgar score}

There were 5262 children with PLIKS, Apgar score and confounders data available, and of these, 33 $(0.6 \%)$ had a score of $\leqslant 6$. Decreasing Apgar score was moderately correlated with resuscitation (Spearman's $\rho=0.32, p<0.001)$. There was little evidence for any increased risk of any suspected or definite PLIKS as Apgar scores decreased (adjusted OR 1.06, 95\% CI $0.95-1.15, p=0.292)$. Evidence of association with reducing Apgar score was stronger when we examined definite PLIKS (adjusted OR 1.30, 95\% CI 1.12-1.50, $p<0.001)$.

\section{Gestational age}

Data on PLIKS, confounders and gestational age in weeks were available for 6004 individuals (mean 39.5, S.D. $=1.8$, range $25-47)$. There were 301 children $(5.0 \%)$ born preterm ( $<37$ weeks) and $455(7.6 \%)$ born postterm ( $>42$ weeks). There was no association between gestational age and any suspected or definite PLIKS in the crude or adjusted analysis (adjusted OR 1.01, $95 \%$ CI $0.96-1.05, p=0.736)$. There was no evidence to support a non-linear (quadratic) relationship with gestational age that might be present if an increased risk of PLIKS were present only at the extremes of gestational age [LRT $\chi^{2}=0.50$, df (1), $p=0.478$ ]. Compared to term births, neither preterm (adjusted OR 0.96, 95\% CI 0.66-1.40) nor post-term (adjusted OR $1.13,95 \%$ CI 0.85-1.52) birth was associated with risk of developing any suspected or definite PLIKS.

\section{Nested sample: diabetes during pregnancy}

There were 1777 children in the nested case-control sample with data available on maternal diabetes, confounders and PLIKS. Of these, 20 mothers (1.1\%) had a diagnosis of diabetes during pregnancy and 11 also had additional evidence of poor blood sugar control. The presence of maternal diabetes was associated with an increased risk of any suspected or definite PLIKS (adjusted OR 2.68, 95\% CI 1.08-6.64, $p=0.034$ ), with a slightly stronger association for definite PLIKS (Table 3). There was a suggestion that the association with any suspected or definite PLIKS was stronger where blood sugar control was poor (adjusted OR $4.41,95 \%$ CI 1.16-16.81) as compared to good (OR $1.56,95 \%$ CI $0.41-5.92)$. However, the CIs were very wide and overlapped substantially, whereas this difference was much less marked for definite PLIKS. Further adjustment for birthweight and IQ score made minimal difference to the results.

\section{Nested sample: pre-eclampsia during pregnancy}

There were 1569 children in the nested case-control sample with data available on maternal pre-eclampsia, PLIKS and confounders. Of these, 33 (2.1\%) had mothers with pre-eclampsia during pregnancy and five of these had evidence of IUGR. Maternal preeclampsia was not associated with risk of any suspected or definite PLIKS in the crude or adjusted analyses (adjusted OR 1.03, 95\% CI 0.50-2.13, $p=$ 0.929). The estimate of association was slightly stronger where there was additional evidence of IUGR (adjusted OR 1.30, 95\% CI 0.21-8.00) compared to where there was no evidence of this (adjusted OR 0.99, 95\% CI 0.45-2.18). However, these estimates were based on small numbers of events, and CIs were wide and overlapped substantially.

\section{Independence of effects}

We included maternal infection during pregnancy, diabetes during pregnancy, and resuscitation all in the same model to examine whether associations for these exposures were independent of one another. In this full model, the estimates of association between each of these exposures and any suspected or definite PLIKS were almost unchanged.

\section{Secondary analyses: frequency of PLIKS and bizarre PLIKS}

There were 165 children (2.6\% of those interviewed) who had definite, frequent (occurring $\geqslant$ monthly) PLIKS, and 233 (3.6\% of those interviewed) rated as having any suspected or definite 'bizarre' PILKS. There was no consistent pattern that associations with the exposures were stronger when examining these more stringent outcomes. 


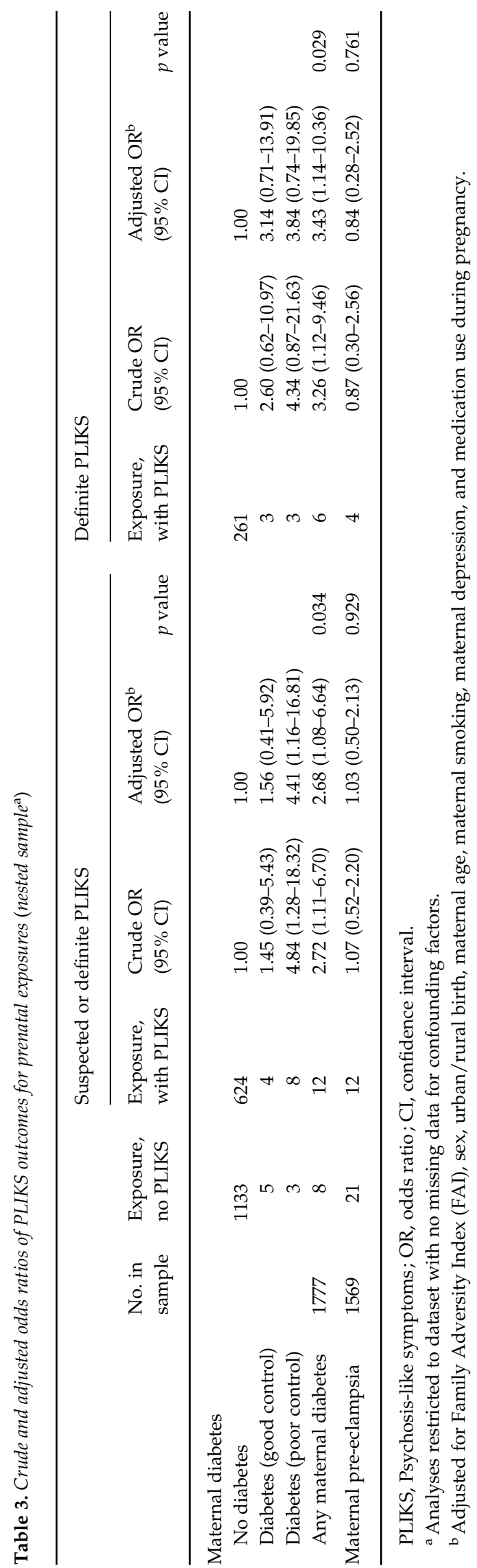

\section{Missing data}

Compared to subjects completing the PLIKSi, those with missing data for PLIKS were more likely to have a history of maternal infection during pregnancy $(55.4 \%$ v. $48.5 \%)$, have been born preterm $(6.4 \% v$. $5.0 \%)$, have a low Apgar score $(1.3 \%$ v. $0.7 \%)$, or have required resuscitation $(8.3 \%$ v. $7.4 \%)$. The results from the multivariable multiple-imputation models were very similar to those using the main dataset, although more precisely estimated, when we imputed confounders only, and also with additional imputation of the outcome measure too.

\section{Discussion \\ Prenatal exposures}

Maternal infection during pregnancy was associated with increased risk of PLIKS, with no evidence that this association was any stronger for influenza compared to other infections. Although the CIs overlapped substantially, and results from subgroup comparisons should be interpreted cautiously, exposure to infection during early pregnancy seemed to be more strongly associated with risk of PLIKS than exposure during late pregnancy. Adjusting for confounders had only a small effect on explaining this association.

We found no evidence that pre-eclampsia was associated with risk of PLIKS, but maternal diabetes during pregnancy was associated with an increased risk of PLIKS in the offspring. The association between diabetes and PLIKS seemed to be stronger where there was evidence of poor glucose control. However, these findings for diabetes and pre-eclampsia are based on only small numbers of women with these exposures, and the robustness of these findings is therefore uncertain.

\section{Perinatal exposures}

There was some evidence that our primary measure of hypoxia, resuscitation, was associated with an increased risk of PLIKS, although evidence for this was not strong. Admission to a neonatal unit following resuscitation is likely to index infants who experienced a greater degree of hypoxia than those not admitted, and indeed estimates of association with PLIKS were larger for such children. We had limited power to investigate this group of infants separately, but infants with encephalopathy did not seem to have a greater risk of PLIKS than infants admitted without neurological signs. It is plausible that a subtle degree of hypoxic damage, insufficient to produce encephalopathy, is nevertheless sufficient to impact upon risk 
of PLIKS. These data are consistent with a continuum of reproductive casualty (Pasamanick et al. 1956), whereby long-term adverse consequences of perinatal hypoxia may occur even in infants without detectable shorter-term neurological sequelae of their hypoxia.

A lower 5-min Apgar score was also associated with risk of definite, but not suspected, PLIKS. Although low Apgar score is often used as a marker of perinatal hypoxia, low Apgar scores are not specific to hypoxia and may be due to other pathologies (ACOG, 2006). Indeed, the correlation between Apgar score and need for resuscitation in our sample was not strong, making it more difficult to postulate possible mechanisms leading to increased risk of PLIKS. We found no evidence of increased risk of PLIKS in pre- or postterm births, even though preterm births in particular have been associated with increased vulnerability to effects of hypoxia and adverse neurological outcomes (Fawke, 2007).

\section{Non-causal explanations}

All of the adverse pre- and perinatal exposures we examined were more common in subjects with evidence of maternal depression and other markers of family adversity during pregnancy. The distribution patterns of other confounders were less consistent across exposures. Although residual confounding can never be eliminated from observational studies, adjusting for confounders only explained a small part of the association with maternal infection during pregnancy, and had a minimal effect on the results for the other exposures.

Bias due to misclassification of data or attrition could also lead to incorrect estimates of association. Misclassification of data is more likely for selfreported data such as infection during pregnancy. Evidence of association with PLIKS was weaker for maternal self-reports of diabetes than for clinician diagnoses obtained from obstetric records (results available on request), which may be indicative of greater misclassification in the self-reported data. However, misclassification of data, if non-differential, leads to underestimates of association, and there is no reason to suppose that misclassification of any exposure data examined was differential with respect to PLIKS status in this cohort.

Although this is a large cohort, with a wealth of detailed information, missing data due to attrition and wave non-response was not insubstantial, a problem common to other large-scale longitudinal studies (Plewis et al. 2004; Callaway et al. 2007). Estimates for all exposures, however, were similar in the multiple-imputation analyses, indicating that attrition is unlikely to have substantially biased these results.

\section{Potential biological mechanisms}

If the associations we observed for maternal infection, maternal diabetes and markers of hypoxia are indeed causal in nature, then it is possible to speculate about possible mechanisms that might underlie them. Associations between maternal infections during pregnancy (serological evidence of infection from a variety of pathogens) and schizophrenia (Brown et al. 2004; Byrne et al. 2007) have been attributed to a variety of possible mechanisms (Cannon et al. 2003) that might also increase risk of PLIKS. These include direct toxic effects of infectious agents on foetal brain development, harmful effects of hyperthermia, or through cytokine production as part of a maternal inflammatory response. Animal studies show that maternal exposure to viral infections during pregnancy can lead to brain gene expression and neuropathology changes in the offspring, and that these changes may vary according to whether exposure occurs early or late during pregnancy (Fatemi et al. 2008). Associations between analgesia use during pregnancy and schizophrenia have also been reported (Sorensen et al. 2004), although adjusting for analgesic use during pregnancy (that was more common in women who reported infections) had no effect on our results.

Hypoxia can lead to cellular damage and death, probably secondary to the development of metabolic acidosis, with vascular watershed areas of the brain within frontal and parietal cortices being particularly susceptible to such damage (Inder et al. 2004). There is an increasing body of evidence that clinically important brain damage can occur even where the hypoxic insult is not significant enough to produce clinical encephalopathy in the early neonatal period. For example, data from the ALSPAC cohort are consistent with hypoxia leading to lower IQ score during childhood even in children without signs of neonatal encephalopathy (Odd et al., unpublished observations).

Adverse effects of dysfunctional glucose metabolism on cerebral development are also plausible. Poorly controlled maternal diabetes has been associated with increased risk of offspring neurodevelopmental impairment (Ornoy, 2005), although how foetal brain development is effected by maternal glucose levels is far from clear at present.

It is perhaps surprising that the associations we observed between PLIKS and maternal infection, resuscitation and depressed Apgar score were not mediated to any degree by childhood IQ score. However, it may be that risk of PLIKS following hypoxic or other 
cellular injury is mediated through more subtle effects than those measurable by testing of IQ score, for example through effects on social cognition, sensory gating or cognitive appraisal.

\section{PLIKS and schizophrenia}

At present, the status of PLIKS in relation to rare clinical disorders such as schizophrenia is not clear. However, our results for PLIKS are reasonably consistent with patterns of associations also reported for schizophrenia in relation to maternal infection during pregnancy (especially early pregnancy), maternal diabetes and markers of perinatal hypoxia (Cannon et al. 2002). All the associations we observed were slightly larger for the narrower outcome of definite PLIKS, but there was no consistent evidence that more frequent symptoms, or specific types of symptoms, indexed stronger associations with the perinatal exposures examined.

\section{Study limitations}

The main limitations of this study relate to potential bias from attrition and misclassification, as discussed above. Furthermore, the exposures we examined are all, to varying extents, simply markers of biological exposures that we were attempting to capture. For example, although it is a strength of our study that we required the presence of positive pressure ventilation or cardiac compressions as our primary measure of hypoxia rather than the more commonly used, but less valid, Apgar score, resuscitation is not a direct measure of whether substantial foetal cellular hypoxia did in fact occur. Similarly, maternal diabetes is unlikely to be a strong marker of foetal exposure to adverse glycaemic levels, even where we attempted to incorporate evidence of poor glucose control, whereas maternal self-rated distinction between influenza as opposed to other infections is also unlikely to reflect the true underlying pathology. Despite these limitations, these results nevertheless have the potential to inform the direction of future studies that aim to assist our understanding of the development of psychotic experiences in the population.

Increasing understanding of PLIKS aetiology is likely to be of substantial importance as PLIKS are so common in population-based samples, and as they have been associated with decreased occupational and social functioning over time (Hanssen et al. 2005; Rossler et al. 2007). Such symptoms might therefore have a large impact on population health and quality of life outside the arena of clinical services, in the same way that depression does.

\section{Conclusion}

Our results are consistent with the hypothesis that adverse biological events during early development may lead to an increased risk of developing psychotic symptoms during childhood. Furthermore, the similarity between these results and findings reported for schizophrenia indicate that future studies of PLIKS may help us to understand how psychotic experiences and clinical disorders develop throughout the lifecourse.

\section{Acknowledgements}

We are extremely grateful to all the families who took part in this study, the midwives for their help in recruiting them, and the whole ALSPAC team, which includes interviewers, computer and laboratory technicians, clerical workers, research scientists, volunteers, managers, receptionists and nurses. The UK Medical Research Council, the Wellcome Trust and the University of Bristol provide core support for ALSPAC. This study was funded by the Wellcome Trust grant no. GR072043MA. Dr Zammit is funded through a Clinician Scientist Award funded by the National Assembly for Wales.

\section{Declaration of Interest}

None.

\section{References}

ACOG (2006). ACOG Committee Opinion: the Apgar score. Obstetrics and Gynecology 107, 1209-1212.

Bowen E, Heron J, Waylen A, Wolke D (2005). Domestic violence risk during and after pregnancy: findings from a British longitudinal study. British Journal of Obstetrics and Gynaecology 112, 1083-1089.

Brown AS, Begg MD, Gravenstein S, Schaefer CA, Wyatt RJ, Bresnahan M, Babulas VP, Susser ES (2004). Serologic evidence of prenatal influenza in the etiology of schizophrenia. Archives of General Psychiatry 61, 774-780.

Byrne M, Agerbo E, Bennedsen B, Eaton WW, Mortensen PB (2007). Obstetric conditions and risk of first admission with schizophrenia: a Danish national register based study. Schizophrenia Research 97, 51-59.

Callaway LK, McIntyre HD, O'Callaghan M, Williams GM, Najman JM, Lawlor DA (2007). The association of hypertensive disorders of pregnancy with weight gain over the subsequent 21 years: findings from a prospective cohort study. American Journal of Epidemiology 166, 421-428.

Cannon M, Jones PB, Murray RM (2002). Obstetric complications and schizophrenia: historical and meta-analytic review. American Journal of Psychiatry 159, 1080-1092. 
Cannon M, Kendell R, Susser E, Jones P (2003). Prenatal and perinatal risk factors for schizophrenia. In The Epidemiology of Schizophrenia (ed. R. Murray, P. B. Jones, E. Susser, J. van Os and M. Cannon), pp. 74-99. Cambridge University Press: Cambridge.

Cox JL, Holden JM, Sagovsky R (1987). Detection of postnatal depression. Development of the 10-item Edinburgh Postnatal Depression Scale. British Journal of Psychiatry 150, 782-786.

Eaton WW, Romanoski A, Anthony JC, Nestadt G (1991). Screening for psychosis in the general population with a self-report interview. Journal of Nervous and Mental Disorders 179, 689-693.

Fatemi SH, Reutiman TJ, Folsom TD, Huang H, Oishi K, Mori S, Smee DF, Pearce DA, Winter C, Sohr R, Juckel G (2008). Maternal infection leads to abnormal gene regulation and brain atrophy in mouse offspring: implications for genesis of neurodevelopmental disorders. Schizophrenia Research 99, 56-70.

Fawke J (2007). Neurological outcomes following preterm birth. Seminars in Fetal and Neonatal Medicine 12, 374-382.

Golding J, Pembrey M, Jones R (2001). ALSPAC - the Avon Longitudinal Study of Parents and Children. I. Study methodology. Paediatric and Perinatal Epidemiology 15, 74-87.

Hanssen M, Bak M, Bijl R, Vollebergh W, van Os J (2005). The incidence and outcome of subclinical psychotic experiences in the general population. British Journal of Clinical Psychology 44, 181-191.

Horwood J, Salvi G, Thomas K, Duffy L, Gunnell D, Hollis C, Lewis G, Menezes P, Thompson A, Wolke D, Zammit S, Harrison G (2008). IQ and non-clinical psychotic symptoms in 12-year-olds: results from the ALSPAC birth cohort. British Journal of Psychiatry 193, 185-191.

Inder T, Neil J, Yoder B, Rees S (2004). Non-human primate models of neonatal brain injury. Seminars in Perinatology 28, 396-404.

Johns LC, Cannon M, Singleton N, Murray RM, Farrell M, Brugha T, Bebbington P, Jenkins R, Meltzer H (2004). Prevalence and correlates of self-reported psychotic symptoms in the British population. British Journal of Psychiatry 185, 298-305.

Kendler KS, Gallagher TJ, Abelson JM, Kessler RC (1996). Lifetime prevalence, demographic risk factors, and diagnostic validity of nonaffective psychosis as assessed in a US community sample. The National Comorbidity Survey. Archives of General Psychiatry 53, 1022-1031.

Ornoy A (2005). Growth and neurodevelopmental outcome of children born to mothers with pregestational and gestational diabetes. Pediatric Endocrinology Reviews 3, 104-113.

Pasamanick B, Rogers ME, Lilienfeld AM (1956). Pregnancy experience and the development of behavior disorders in children. American Journal of Psychiatry 112, 613-618.

Perala J, Suvisaari J, Saarni SI, Kuoppasalmi K, Isometsa E, Pirkola S, Partonen T, Tuulio-Henriksson A, Hintikka J,
Kieseppa T, Harkanen T, Koskinen S, Lonnqvist J (2007). Lifetime prevalence of psychotic and bipolar I disorders in a general population. Archives of General Psychiatry 64, 19-28.

Plewis I, Calderwoof L, Hawkes D, Nathan G (2004). National Child Development Study and 1970 British Cohort Study Technical Report: changes in the NCDS and BCS70 populations and samples over time. Centre for Longitudinal Studies, Institute of Education: London.

Poulton R, Caspi A, Moffitt TE, Cannon M, Murray R, Harrington H (2000). Children's self-reported psychotic symptoms and adult schizophreniform disorder: a 15-year longitudinal study. Archives of General Psychiatry 57, 1053-1058.

Raghunathan TE, Lepkowski JM, Van Hoewyk J, Solenberger P (2001). A multivariate technique for multiply imputing missing values using a sequence of regression models. Survey Methodology 27, 85-95.

Rossler W, Riecher-Rossler A, Angst J, Murray R, Gamma A, Eich D, van Os J, Gross VA (2007). Psychotic experiences in the general population: a twenty-year prospective community study. Schizophrenia Research 92, 1-14.

Royston P (2004). Multiple imputation of missing values. Stata Journal 3, 227-241.

Shaffer D, Fisher P, Lucas CP, Dulcan MK, Schwab-Stone ME (2000). NIMH Diagnostic Interview Schedule for Children Version IV (NIMH DISC-IV) : description, differences from previous versions, and reliability of some common diagnoses. Journal of the American Academy of Child and Adolescent Psychiatry 39, 28-38.

Sorensen HJ, Mortensen EL, Reinisch JM, Mednick SA (2004). Association between prenatal exposure to analgesics and risk of schizophrenia. British Journal of Psychiatry 185, 366-371.

Spauwen J, Krabbendam L, Lieb R, Wittchen HU, van Os J (2004). Early maternal stress and health behaviours and offspring expression of psychosis in adolescence. Acta Psychiatrica Scandinavica 110, 356-364.

St Clair D, Xu M, Wang P, Yu Y, Fang Y, Zhang F, Zheng X, Gu N, Feng G, Sham P, He L (2005). Rates of adult schizophrenia following prenatal exposure to the Chinese famine of 1959-1961. Journal of the American Medical Association 294, 557-562.

Susser E, Neugebauer R, Hoek HW, Brown AS, Lin S, Labovitz D, Gorman JM (1996). Schizophrenia after prenatal famine. Further evidence. Archives of General Psychiatry 53, 25-31.

Thomas K, Harrison G, Zammit S, Lewis G, Horwood J, Heron J, Hollis C, Wolke D, Thompson A, Gunnell D (in press). The association of measures of fetal and childhood growth with non-clinical psychotic symptoms in 12 year olds: the ALSPAC cohort. British Journal of Psychiatry.

van Os J, Hanssen M, Bijl RV, Vollebergh W (2001). Prevalence of psychotic disorder and community level of psychotic symptoms: an urban-rural comparison. Archives of General Psychiatry 58, 663-668. 
Wechsler D (1991). Manual for the Wechsler Intelligence Scale for Children - Third Edition (WISC-III). Psychological Corporation: San Antonio, TX.

WHO (1994). Schedules for Clinical Assessment in Neuropsychiatry. American Psychiatric Research: Washington, DC.
Wiles NJ, Zammit S, Bebbington P, Singleton N, Meltzer H, Lewis G (2006). Self-reported psychotic symptoms in the general population: results from the longitudinal study of the British National Psychiatric Morbidity Survey. British Journal of Psychiatry $\mathbf{1 8 8}$

519-526. 